\title{
Editorial
}

\section{BCK-Algebras and Related Algebraic Systems}

\author{
Young Bae Jun, ${ }^{1}$ Ivan Chajda, ${ }^{2}$ Hee Sik Kim, ${ }^{3}$ Eun Hwan Roh, ${ }^{4}$ \\ Jianming Zhan, ${ }^{5}$ and Afrodita Iorgulescu ${ }^{6}$ \\ ${ }^{1}$ Department of Mathematics Education, Gyeongsang National University, \\ Chinju 660-701, Republic of Korea \\ ${ }^{2}$ Department of Algebra and Geometry, Faculty of Sciences, Palacky University (UP), \\ 77147 Olomouc, Czech Republic \\ ${ }^{3}$ Department of Mathematics, Hanyang University, Seoul 133-791, Republic of Korea \\ ${ }^{4}$ Department of Mathematics Education, Chinju National University of Education, \\ Chinju 660-756, Republic of Korea \\ ${ }^{5}$ Department of Mathematics, Hubei Institute for Nationalities, Enshi, Hubei 445000, China \\ ${ }^{6}$ Department of Computer Science, The Bucharest Academy of Economic Studies, \\ 010374 Bucharest, Romania
}

Correspondence should be addressed to Young Bae Jun, skywine@gmail.com

Received 5 November 2011; Accepted 10 November 2011

Copyright (C) 2011 Young Bae Jun et al. This is an open access article distributed under the Creative Commons Attribution License, which permits unrestricted use, distribution, and reproduction in any medium, provided the original work is properly cited.

BCK/BCI-algebras are algebraic structures, introduced by K. Iséki in 1966, that describe fragments of the propositional calculus involving implication known as BCK/BCI-logics. It is known that the class of BCK-algebras is a proper subclass of the class of BCI-algebras. We refer the reader for useful textbooks for BCK/BCI-algebra to [1-3].

The aim of this special issue was to promote the exchange of ideas between researchers and to spread new trends in this area. It is focused on all aspects of BARAS, from their foundations to applications in computer sciences and informatics.

This special issue contains nine papers. In the paper entitled "Commutative pseudo valuations on BCK-algebras," M. I. Doh and M. S. Kang introduced the notion of a commu-tative pseudo valuation on a BCK-algebra and investigated its characterizations. They discussed the relationship between a pseudo valuation and a commutative pseudo valua-tion. They also provided conditions for a pseudo valuation to be a commutative pseudo valuation.

Neggers et al. [4] introduced the notion of $Q$-algebras which are a generalization of $\mathrm{BCK} / \mathrm{BCI} / \mathrm{BCH}$-algebras, obtained several properties, and discussed quadratic $Q$-algebras. In the paper, entitled "A construction of mirror $Q$-algebras," K. S. So introduced the notion of mirror algebras to $Q$-algebras, and she investigated how to construct mirror $Q$-algebras 
from a $Q$-algebra. She also obtained the necessary conditions for a left mirror algebra $(M(X), *,(0,0))$ of $(X, *, 0)$ to be a $Q$-algebra.

Many authors studied the graph theory in connection with (commutative) semigroups and (commutative and noncommutative) rings as shown in the references that we refer the reader to. For example, Beck [5] associated to any commutative ring $R$ its zero-divisor graph $G(R)$ whose vertices are the zero-divisors of $R$ (including 0 ), with two vertices $a, b$ joined by an edge in case $a b=0$. Also, DeMeyer et al. [6] defined the zero-divisor graph of a commutative semigroup $S$ with zero $(0 x=0 \forall x \in S)$. Motivated by these works, in the paper, entitled "Graphs based on BCK/BCI-algebras,", Y. B. Jun and K. S. Lee studied graph theory based on BCK-algebras. They tried to discuss the associated graphs of BCK/BCI-algebras. To do so, they first introduced the notions of (l-prime) quasi-ideals and zero-divisors and investigated related properties. They introduced the concept of associative graph of a BCK/BCI-algebra and provided several examples. They provided conditions for a proper (quasi)ideal of a $\mathrm{BCK} / \mathrm{BCI}$-algebra to be $l$-prime. Finally they showed that the associative graph of a BCKalgebra is a connected graph in which every nonzero vertex is adjacent to 0 , but the associative graph of a BCI-algebra is not connected by providing an example.

In the paper, entitled, "Fuzzy filter spectrum of a BCK algebra," X. L. Xin et al. investigated both the topological structure and fuzzy structure on BCK-algebras. They introduced the concept of fuzzy s-prime filters and discussed some related properties. Using the fuzzy sprime filters, they established a fuzzy topological structure on bounded commutative BCKalgebras and bounded implicative BCK-algebras, respectively.

In the paper, entitled "Generalized derivations and bilocal jordan derivations of nest algebras," D. Yan and C. Zhang discussed generalized and bilocal Jordan derivations of nest algebras. They showed that in nest algebra,

(1) (bi)local Jordan derivations are inner derivations and,

(2) generalized derivations are generalized inner derivations.

In the paper, entitled "On algebraic approach in quadratic systems," M. Mencinger considered homogeneous quadratic systems via the so-called Markus approach. He used the one-to-one correspondence between homogeneous quadratic dynamical systems and algebra which was originally introduced by Markus in [7] and considered some general connections and the influence of power associativity in the corresponding quadratic system.

In [8], H. S. Kim and Y. H. Kim introduced the notion of a BE-algebra as a generalization of a BCK-algebra and gave an equivalent condition of the filter in $B E$-algebras by using the notion of upper sets. In $[9,10], \mathrm{Ahn}$ and So introduced the notion of ideals in $B E$-algebras and proved several characterizations of such ideals. In the paper, entitled "On $B E$-semigroups," S. S. Ahn and Y. H. Kim combined BE-algebras and semigroups to introduce the notion of $B E$-semigroups. They defined left (resp., right) deductive systems ((LDS, resp., RDS) for short) of a $B E$-semigroup, and then they described LDS generated by a nonempty subset in a $B E$-semigroup as a simple form.

In the paper entitled "Two new types of rings constructed from quasiprime ideals," M. Ghanem and H. Al-Ezeh generalized the well-known concepts of regular and PF-rings to ordinary differential rings and considered well-known properties of regular and PF-rings in their situations.

In the paper entitled "Rough filters in BL-algebras," L. Torkzadeh and S. Ghorbani applied the rough set theory to $B L$-algebras, and introduced the notion of rough filters (subalgebras) of $B L$-algebras as a generalization of filters (subalgebras) of $B L$-algebras. 
Although the selected topics and papers are not an exhaustive representation of the area of BARASs, they present the rich and many-faceted knowledge that we have the pleasure of sharing with the readers.

\section{Acknowledgments}

It was our honor to receive submissions from many authors. We would like to thank the authors for their excellent contributions and patience in assisting us. Moreover, the fundamental work of all reviewers on these papers is also very warmly acknowledged. Last, but not least, the publishing staff have worked diligently with us on this special issue. We also would like to express our sincere gratitude to the publishing staff.

Young Bae Jun Ivan Chajda

Hee Sik Kim

Eun Hwan Roh

Jianming Zhan

Afrodita Iorgulescu

\section{References}

[1] Y. S. Huang, BCI-Algebra, Science Press, Beijing, China, 2006.

[2] A. Iorgulescu, Algebras of Logic as BCK Algebras, Editura ASE, Bucharest, Romania, 2008.

[3] J. Meng and Y. B. Jun, BCK-Algebras, Kyung Moon Sa Co., Seoul, Korea, 1994.

[4] J. Neggers, S. S. Ahn, and H. S. Kim, "On Q-algebras," International Journal of Mathematics and Mathematical Sciences, vol. 27, no. 12, pp. 749-757, 2001.

[5] I. Beck, "Coloring of commutative rings," Journal of Algebra, vol. 116, no. 1, pp. 208-226, 1988.

[6] F. R. DeMeyer, T. McKenzie, and K. Schneider, "The zero-divisor graph of a commutative semigroup," Semigroup Forum, vol. 65, no. 2, pp. 206-214, 2002.

[7] L. Markus, "Quadratic differential equations and non-associative algebras," vol. 45 of Annals of Mathematics Studies, Princeton University Press, Princeton, NJ, USA, 1960, pp. 185-213.

[8] H. S. Kim and Y. H. Kim, "On BE-algebras," Scientiae Mathematicae Japonicae, vol. 66, no. 1, pp. 113-116, 2007.

[9] S. S. Ahn and K. S. So, "On ideals and upper sets in BE-algebras," Scientiae Mathematicae Japonicae, vol. 68, no. 2, pp. 279-285, 2008.

[10] S. S. Ahn and K. S. So, "On generalized upper sets in BE-algebras," Bulletin of the Korean Mathematical Society, vol. 46, no. 2, pp. 281-287, 2009. 


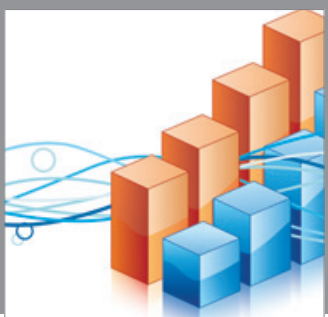

Advances in

Operations Research

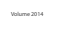

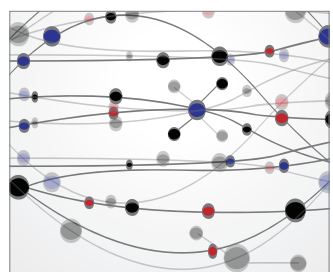

\section{The Scientific} World Journal
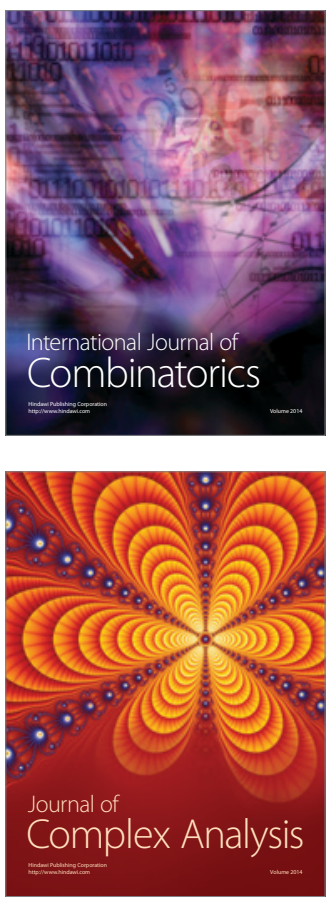

International Journal of

Mathematics and

Mathematical

Sciences
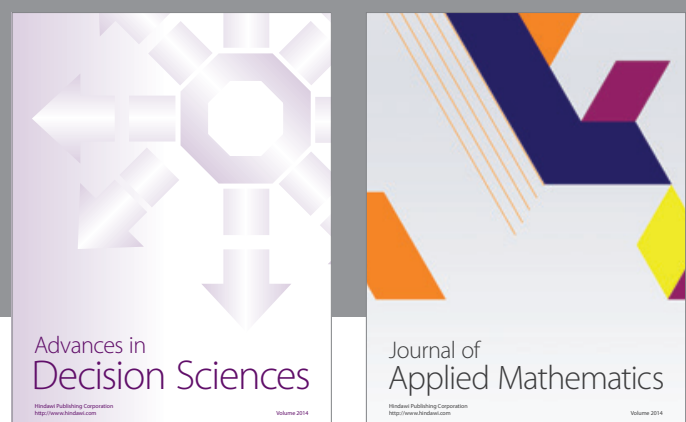

Journal of

Applied Mathematics
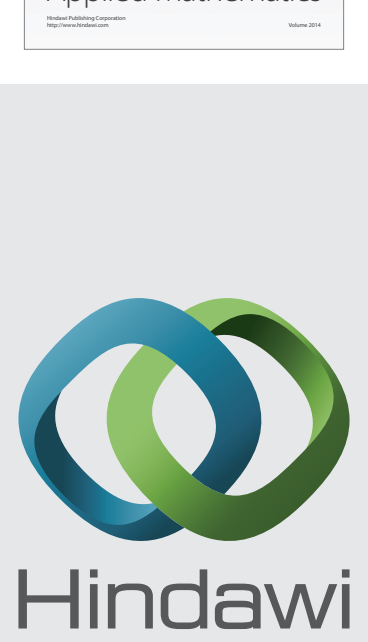

Submit your manuscripts at http://www.hindawi.com
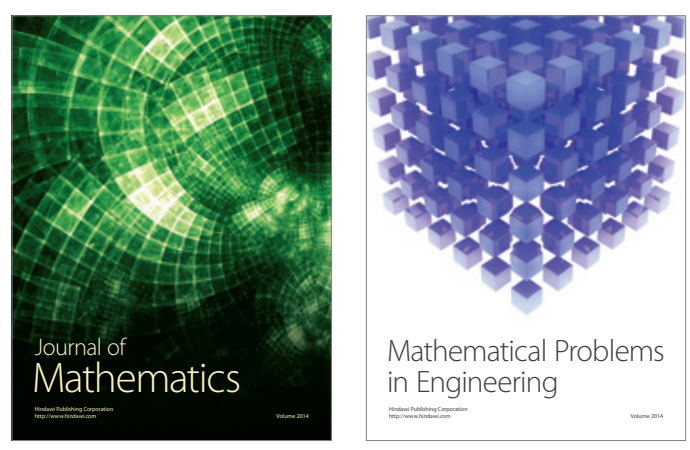

Mathematical Problems in Engineering
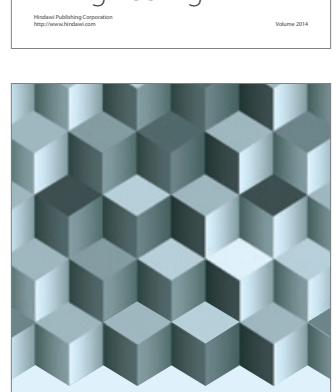

Journal of

Function Spaces
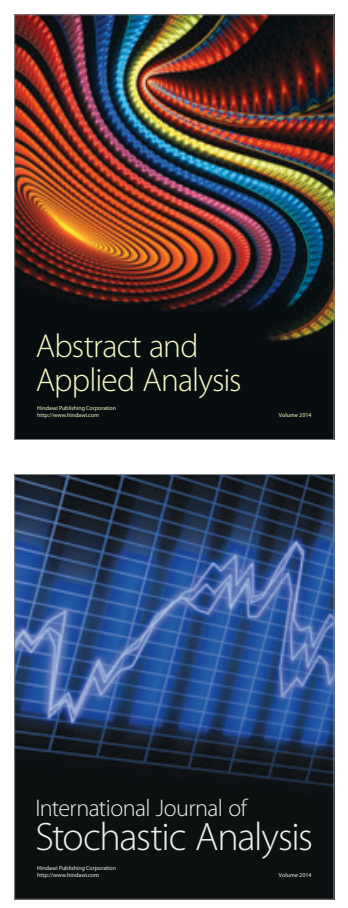

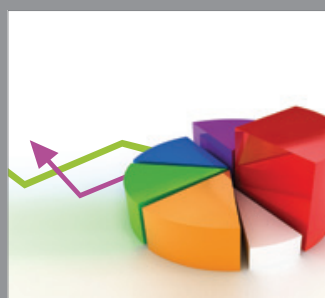

ournal of

Probability and Statistics

Promensencen
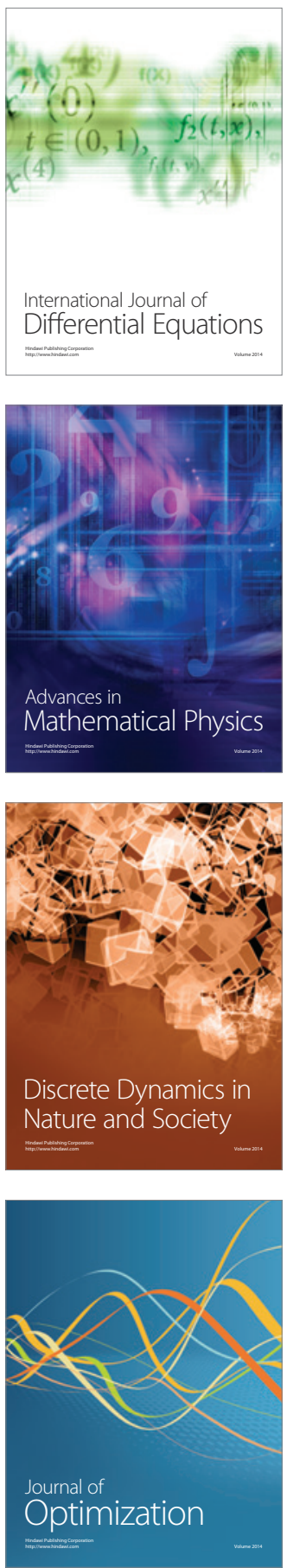